\title{
No evidence of a causal association of type 2 diabetes and glucose metabolism with atrial fibrillation
}

\author{
Hadi Harati ${ }^{1}$ - Daniela Zanetti ${ }^{2}$ - ${\text { Abhiram } \text { Rao }^{3} \text { - Stefan Gustafsson }}^{4} \cdot$ Marco Perez $^{2}$ - Erik Ingelsson ${ }^{2,5,6}$. \\ Joshua W. Knowles ${ }^{2,5,6}$
}

Received: 6 September 2018 / Accepted: 29 January 2019 / Published online: 27 February 2019

(C) Springer-Verlag GmbH Germany, part of Springer Nature 2019

\begin{abstract}
Aims/hypothesis Several epidemiological studies have shown an increased risk of atrial fibrillation in individuals with type 2 diabetes or milder forms of dysglycaemia. We aimed to assess whether this relation is causal using a Mendelian randomisation approach.

Methods Two-sample Mendelian randomisation was used to obtain estimates of the influence of type 2 diabetes, fasting blood glucose $(\mathrm{FBG})$, and $\mathrm{HbA}_{1 \mathrm{c}}$ on the risk of atrial fibrillation. Instrumental variables were constructed using available summary statistics from meta-analyses of genome-wide association studies (GWAS) for type 2 diabetes and associated phenotypes. Pleiotropic SNPs were excluded from the analyses. The most recent GWAS meta-analysis summary statistics for atrial fibrillation, which included over 1 million individuals (approximately 60,000 individuals with atrial fibrillation) was used for outcome analysis.

Results Neither type 2 diabetes (OR 1.01 [95\% CI 0.98, 1.03]; $p=0.37$ ), nor FBG (OR 0.95 [95\% CI 0.82, 1.09] per mmol/l; $p=$ $0.49)$ or $\mathrm{HbA}_{1 \mathrm{c}}(\mathrm{OR} 1.01$ [95\% CI, $0.85,1.17$ ] per $\mathrm{mmol} / \mathrm{mol}[\%] ; p=0.88)$ were associated with atrial fibrillation in Mendelian randomisation analyses. We had $>80 \%$ statistical power to detect ORs of 1.08, 1.06 and 1.09 or larger for type 2 diabetes, FBG and $\mathrm{HbA}_{1 \mathrm{c}}$, respectively, for associations with atrial fibrillation.

Conclusions/interpretation This Mendelian randomisation analysis does not support a causal role of clinical significance between genetically programmed type 2 diabetes, $\mathrm{FBG}$ or $\mathrm{HbA}_{1 \mathrm{c}}$ and development of atrial fibrillation. These data suggest that drug treatment to reduce dysglycaemia is unlikely to be an effective strategy for atrial fibrillation prevention.

Data availability The datasets analysed during the current study are available from the following repository: Nielsen JB, Thorolfsdottir RB, Fritsche LG, et al (2018) GWAS summary statistics for AF ( $N=60,620$ AF cases and 970,216 controls). Center for Statistical Genetics: http://csg.sph.umich.edu/willer/public/afib2018/nielsen-thorolfsdottir-willer-NG2018-AFibgwas-summary-statistics.tbl.gz
\end{abstract}

Keywords Atrial fibrillation $\cdot$ Genome-wide association $\cdot$ Mendelian randomisation $\cdot$ Type 2 diabetes

Erik Ingelsson and Joshua W. Knowles contributed equally to this study.

Electronic supplementary material The online version of this article (https://doi.org/10.1007/s00125-019-4836-y) contains peer-reviewed but unedited supplementary material, which is available to authorised users.

Joshua W. Knowles

knowlej@stanford.edu

1 Department of Medicine, Division of Endocrinology, Stanford University School of Medicine, Stanford, CA, USA

2 Department of Medicine, Division of Cardiovascular Medicine, Falk Cardiovascular Research Center, Stanford University School of Medicine, 300 Pasteur Drive, CV 273, Stanford, CA 94305, USA
3 Department of Bioengineering, Stanford University, Stanford, CA, USA

4 Department of Medical Sciences, Molecular Epidemiology and Science for Life Laboratory, Uppsala University, Uppsala, Sweden

5 Stanford Cardiovascular Institute, Stanford University, Stanford, CA, USA

6 Stanford Diabetes Research Center, Stanford University, Stanford, CA, USA 


\section{Research in context}

\section{What is already known about this subject?}

- Type 2 diabetes and milder forms of dysglycaemia are associated with increased risk of incident atrial fibrillation in epidemiological studies

What is the key question?

- Is the association between type 2 diabetes and atrial fibrillation causal?

\section{What are the new findings?}

- We did not observe a causal role between genetically programmed type 2 diabetes, fasting blood glucose or $\mathrm{HbA}_{1 \mathrm{c}}$ and the development of atrial fibrillation

How might this impact on clinical practice in the foreseeable future?

- These data suggest that drug treatment to reduce dysglycaemia is unlikely to be an effective strategy for atrial fibrillation prevention

$\begin{array}{ll}\text { Abbreviations } & \\ \text { AFGen } & \text { Atrial Fibrillation Genetics (Consortium) } \\ \text { FBG } & \text { Fasting blood glucose } \\ \text { GWAS } & \begin{array}{l}\text { Genome-wide association studies } \\ \text { 2hBG }\end{array} \\ \text { MR h blood glucose } \\ \text { MR-PRESSO } & \begin{array}{l}\text { Mendelian randomisation } \\ \text { pleiotropy residual sum and outlier }\end{array}\end{array}$

\section{Introduction}

Atrial fibrillation affects over five million Americans and is a major cause of stroke-related disability and mortality. Interventions to prevent the development of atrial fibrillation or the sequelae of this condition, such as timely initiation of anti-coagulant therapy, are urgently needed. There is strong epidemiological evidence of an independent association between type 2 diabetes and atrial fibrillation, with a $40 \%$ increase in risk of incident atrial fibrillation in individuals with type 2 diabetes after adjusting for major confounders, including obesity [1]. On the other hand, intensive glycaemic control in people with type 2 diabetes did not affect the rate of newonset atrial fibrillation [2]. Compared with a fasting blood glucose (FBG) of $<5.5 \mathrm{mmol} / \mathrm{l}$, an FBG of $5.5-6.9 \mathrm{mmol} / \mathrm{l}$ increased the risk of incident atrial fibrillation by $16 \%$, while an $11 \mathrm{mmol} / \mathrm{mol}(1 \%)$ increase in $\mathrm{HbA}_{1 \mathrm{c}}$ increased this risk by 13\% [3]. The evidence for an association between insulin resistance and atrial fibrillation is more controversial; the metabolic syndrome increases the risk of incident atrial fibrillation by $67 \%$, whereas fasting insulin has generally not been shown to increase this risk [4].

Although these studies have identified type 2 diabetes and its related phenotypes as independent risk factors for atrial fibrillation, their results are not conclusive and may be confounded by other abnormalities that cluster with type 2 diabetes, including obesity and hypertension. Therefore, causality has not been established. Mendelian randomisation is a study design in which random assignment of an individual's genetic variants at conception are employed as instrumental variables for estimating the unconfounded causal effect of a risk factor on a disease. In this study, we used Mendelian randomisation techniques to test, for the first time, the hypothesis that the known positive observational relationship between type 2 diabetes and its related phenotypes and incident atrial fibrillation is causal.

\section{Methods}

Study population To obtain information on the genetic variants associated with type 2 diabetes, a recent meta-analysis of genome-wide association data from $\sim 160,000$ individuals of European ancestry were used [5]. The Meta-Analysis of Glucose and Insulin-related traits Consortium (MAGIC) [6] was used to determine the variants associated with type 2 diabetes-related traits, including FBG, 2 h blood glucose (2hBG), $\mathrm{HbA}_{1 \mathrm{c}}$ and fasting insulin.

For outcome analyses, we used the latest meta-analysis of GWAS for atrial fibrillation, which compared a total of 60,620 individuals with atrial fibrillation with 970,216 control individuals of European ancestry from six contributing studies, including the Nord-Trøndelag Health Study (HUNT), the Diabetes Epidemiology: Collaborative analysis of Diagnostic criteria in Europe study (DECODE), the Michigan Genomics Initiative (MGI), DiscovEHR, UK Biobank, and the Atrial Fibrillation Genetics (AFGen) Consortium [7].

Statistical analysis We performed two-sample Mendelian randomisation analysis to determine the causal relationships of type 2 diabetes, FBG, $2 \mathrm{hBG}, \mathrm{HbA}_{1 \mathrm{c}}$ and fasting insulin 
with atrial fibrillation using the TwoSampleMR package in $\mathrm{R}$ (v0.4.18; https://mrcieu.github.io/TwoSampleMR/). Instrumental variables were initially constructed in three steps; first, the SNPs associated with type 2 diabetes or related phenotypes at GWAS significance $\left(p<5 \times 10^{-8}\right)$ were selected. Second, the SNPs that were in significant linkage disequilibrium (LD) with one another $\left(R^{2}>0.1\right.$; calculated by the online tool rAggr [http://raggr.usc.edu/. Accessed 11 January 2018] using the European reference population) were excluded. Third, the pleiotropic SNPs were excluded as outlined below (ESM Fig. 1).

We used three methods for the Mendelian randomisation analysis. The inverse-variance weighted method is a fixedeffect meta-analysis of the causal estimates from each of the genetic variants. However, if there was heterogeneity between the causal estimates of different variants indicating the presence of pleiotropy, Egger regression or weighted median were more appropriate for use [8]. For sensitivity analysis, we used the Mendelian randomisation pleiotropy residual sum and outlier (MR-PRESSO) test to identify horizontal pleiotropic outliers in multi-instrument summarylevel Mendelian randomisation testing [9]. The Bonferroni corrected $p$ value of 0.05 divided by the number of variants was used to find the outliers. We then evaluated the extent to which outliers caused distortion in the causal estimates by comparing the causal estimates from the inverse-variance weighted meta-analysis before and after removal of outlier variants detected by the MR-PRESSO outlier test. The intercept term in the Mendelian randomisation-Egger (MREgger) regression analysis was used to further test for directional horizontal pleiotropy.

Power calculations for Mendelian randomisation were performed according to the formula proposed by Burgess [10]. We estimated the minimum effect size to be able to observe the measured outcome (atrial fibrillation) using a total sample size of 1,030,836 with 60,620 cases of atrial fibrillation, an $\alpha$ threshold of 0.05 , variance explained for each specific instrumental variable, and power $>80 \%$. A fixed effect size of $10 \%$ (OR 1.10) was arbitrarily set as clinically significant. The variability explained by each instrumental variable was calculated by the TwoSampleMR package in $\mathrm{R}$ using the MR Steiger directionality test.

\section{Results}

Our instrumental variables for $2 \mathrm{hBG}$ and fasting insulin showed an explained variance of $0.65 \%$ and $0.50 \%$, respectively, resulting in limited statistical power with a minimum detectable OR of 1.17 for atrial fibrillation, which was higher than our preset fixed effect size for clinical relevance. Therefore, we decided not to proceed with Mendelian randomisation analyses for these phenotypes. We had $>80 \%$ power to detect effect sizes of at least $1.08,1.06$ and 1.09 for type 2 diabetes, $\mathrm{FBG}$ and $\mathrm{HbA}_{1 \mathrm{c}}$, respectively (all below the predefined threshold of 1.10 for clinical relevance).

The MR-PRESSO method identified 4, 1 and 0 pleiotropic SNPs for type 2 diabetes, $\mathrm{FBG}$ and $\mathrm{HbA}_{1 \mathrm{c}}$ respectively (ESM Fig. 1). Table 1 shows the results of Mendelian randomisation analysis for type 2 diabetes, FBG and $\mathrm{HbA}_{1 \mathrm{c}}$. The calculated ORs $(95 \% \mathrm{CI})$ for atrial fibrillation after exclusion of these SNPs were $1.01(0.98,1.03 ; p=0.37)$ for type 2 diabetes, 0.95 $(0.82,1.09 ; p=0.49)$ for FBG and $1.01(0.85,1.17 ; p=0.88)$ for $\mathrm{HbA}_{1 \mathrm{c}}$. Comparing the causal estimates from the MRPRESSO meta-analysis before and after removal of outlier variants showed no evidence of distortion in the estimates ( $p=0.68, p=0.91$ and $p=0.88$, respectively, for type 2

Table 1 No evidence of a causal association of genetic variants associated with type 2 diabetes, fasting glucose and $\mathrm{HbA}_{1 \mathrm{c}}$ with atrial fibrillation using Mendelian randomisation analysis

\begin{tabular}{|c|c|c|c|c|c|c|c|c|c|c|c|}
\hline \multirow[t]{3}{*}{ IV } & \multirow[t]{3}{*}{ No. of SNPs } & \multicolumn{6}{|c|}{ Mendelian randomisation method used } & \multirow{2}{*}{\multicolumn{2}{|c|}{ Pleiotropy test }} & \multirow{3}{*}{$\begin{array}{l}\text { Variability } \\
\text { explained by } \\
\mathrm{IV}^{\mathrm{b}}(\%)\end{array}$} & \multirow{3}{*}{$\begin{array}{l}\text { Minimum detectable } \\
\text { OR for AF with } \\
>80 \% \text { power }^{\mathrm{c}}\end{array}$} \\
\hline & & \multicolumn{2}{|l|}{ MR-Egger } & \multicolumn{2}{|l|}{ Weighted median } & \multicolumn{2}{|l|}{ MR-PRESSO } & & & & \\
\hline & & $\begin{array}{l}\mathrm{AF} \mathrm{OR}{ }^{\mathrm{a}} \\
(95 \% \mathrm{CI})\end{array}$ & $p$ & $\begin{array}{l}\mathrm{AF} \mathrm{OR}^{\mathrm{a}} \\
(95 \% \mathrm{CI})\end{array}$ & $p$ & $\begin{array}{l}\mathrm{AF} \mathrm{OR}^{\mathrm{a}} \\
(95 \% \mathrm{CI})\end{array}$ & $p$ & $\begin{array}{l}\text { Egger } \\
\text { intercept }\end{array}$ & $p$ & & \\
\hline $\mathrm{T} 2 \mathrm{D}$ & 122 & $1.02(0.95,1.09)$ & 0.48 & $1.01(0.98,1.04)$ & 0.07 & $1.01(0.98,1.03)$ & 0.37 & 0.006 & 0.81 & 2.3 & 1.08 \\
\hline FBG & 36 & $1.07(0.82,1.33)$ & 0.59 & $0.93(0.78,1.08)$ & 0.37 & $0.95(0.82,1.09)$ & 0.49 & -0.004 & 0.28 & 4.5 & 1.06 \\
\hline $\mathrm{HbA}_{1 \mathrm{c}}$ & 11 & $0.76(0.39,1.13)$ & 0.18 & $0.95(0.76,1.13)$ & 0.55 & $1.01(0.85,1.17)$ & 0.88 & 0.012 & 0.13 & 2.1 & 1.09 \\
\hline
\end{tabular}

${ }^{\mathrm{a}}$ The OR for atrial fibrillation were calculated per $1 \mathrm{SD}$ increase in continuous traits ( $\mathrm{mmol} / \mathrm{l}$ for $\mathrm{FBG}$ and $\mathrm{mmol} / \mathrm{mol}[\%]$ for $\mathrm{HbA} \mathrm{A}_{1 \mathrm{c}}$ ) or for having type 2 diabetes

${ }^{\mathrm{b}}$ Calculated using the TwoSampleMR package in $\mathrm{R}$

${ }^{\mathrm{c}}$ The minimum OR for atrial fibrillation was calculated per $1 \mathrm{SD}$ increase in continuous traits or for having type 2 diabetes to be able to observe the measure outcome (atrial fibrillation) with a sample size of $1,038,836$ at an $\alpha$ of 0.05 and power $>80 \%$

AF, atrial fibrillation; IV, instrumental variable; MR-Egger, Mendelian randomisation-Egger; T2D, type 2 diabetes 
diabetes, $\mathrm{FBG}$ and $\mathrm{HbA}_{1 \mathrm{c}}$; data not shown). Egger regression intercept analysis also did not show evidence of pleiotropy in the instrumental variables used for type 2 diabetes, FBG or $\mathrm{HbA}_{1 \mathrm{c}}$ (Table 1). SNP-exposure and SNP-outcome associations are presented in ESM Tables 1-3.

\section{Discussion}

In this Mendelian randomisation-based study using GWAS summary statistics, no causal association was found between genetically determined type 2 diabetes, FBG or $\mathrm{HbA}_{1 \mathrm{c}}$ and atrial fibrillation in individuals of European descent.

The association between type 2 diabetes and incident atrial fibrillation in the general population is mainly observed in people with diagnosed type 2 diabetes [3], which suggests that it is the long-term exposure to hyperglycaemia that may increase the risk of atrial fibrillation. While this and other mechanisms for this association have been hypothesised, there has been little supportive functional data. However, our results show that genetically programmed chronic hyperglycaemia or type 2 diabetes is not associated with atrial fibrillation, indicating that these disturbances in glucose metabolism are not causative of atrial fibrillation. One possibility is that the associated comorbidities of type 2 diabetes, namely hypertension and obesity, are the causal link between type 2 diabetes and atrial fibrillation. In fact, a recent Mendelian randomisation analysis showed a causal relationship between BMI and incident atrial fibrillation [11].

Along with the recent evidence suggesting a causal link between BMI and atrial fibrillation, our results suggest that to prevent atrial fibrillation, it is more important to control comorbidities, such as obesity, than blood glucose levels. In fact, the results of the Action to Control Cardiovascular Risk in Diabetes (ACCORD) trial showed that intensive glycaemic control in people with type 2 diabetes $\left(\mathrm{HbA}_{1 \mathrm{c}}<42 \mathrm{mmol} / \mathrm{mol}\right.$ [6.0\%] vs 53-64 $\mathrm{mmol} / \mathrm{mol}[7.0-7.9 \%]) \mathrm{did}$ not affect the rate of new-onset atrial fibrillation [2]. On the other hand, intensive weight loss through bariatric surgery is associated with lower incidence of atrial fibrillation among individuals with severe obesity [12]. A recent Mendelian randomisation study did not find a causal association between type 2 diabetes and cardiometabolic stroke, a major atrial fibrillation comorbidity [13], which is further evidence that glycaemic control in people with type 2 diabetes may not be a good strategy to reduce the burden of atrial fibrillation in the community.

The main strengths of our investigation include the good level of power to investigate our hypothesis, which was achieved by using the latest GWAS meta-analysis for atrial fibrillation. We also used several methods of Mendelian randomisation analyses, which yielded consistent effects for the tested hypothesis.
Our study also had some limitations. While we had enough power to evaluate the relationship between type 2 diabetes, FPG and $\mathrm{HbA}_{1 \mathrm{c}}$ with atrial fibrillation, our study was limited by lower power to test the same causal associations for fasting insulin and $2 \mathrm{hBG}$. In the AFGen Consortium, atrial fibrillation was defined by ECG in addition to ICD codes, which was different from the other cohorts in the meta-analysis, which used only ICD codes. However, there was very small heterogeneity of effect size across the six contributing studies. The use of summary-level data from a meta-analysis of glycaemic traits, where individuals were genotyped with an array enriched for cardiometabolic variants (the 'Metabochip'), increases the chance of including pleiotropic SNPs [14]. However, this limitation must be balanced by the fact that use of data solely from GWAS that did not employ Metabochip-derived results would have resulted in a study with significantly lower power. Finally, our study included participants of European ancestry and, therefore, our findings may not be generalisable to non-European individuals.

In conclusion, in this current Mendelian randomisation study of more than one million individuals of European ancestry, no causal relationship was found between type 2 diabetes or dysglycaemia and risk of atrial fibrillation. These data, along with the previously reported causal link between BMI and atrial fibrillation, suggest that to combat the expanding global burden of atrial fibrillation, control of type 2 diabetes-related comorbidities (like obesity) is of more value than glycaemic control.

Data availability The datasets analysed during the current study are available from the following repository: Nielsen JB, Thorolfsdottir RB, Fritsche LG, et al (2018) GWAS summary statistics for AF $(N=60,620$ AF cases and 970,216 controls). Center for Statistical Genetics: http://csg. sph.umich.edu/willer/public/afib2018/nielsen-thorolfsdottir-willerNG2018-AFib-gwas-summary-statistics.tbl.gz

Funding The research was performed with support from National Institutes of Health (T32DK007217; 1R01HL135313; 1R01DK106236) and the Doris Duke Charitable Foundation. EI and JK were supported by the Stanford Diabetes Research Center (P30DK116074).

Duality of interest The authors declare that there is no duality of interest associated with this manuscript.

Contribution statement $\mathrm{HH}$ contributed to design, analysed and interpreted the data and drafted the manuscript. DZ, AR and SG helped with data analysis and revised the article critically for important intellectual content. MP helped with the interpretation of data and critically revised the article for intellectual content. EI and JK contributed to the design, supervised the work and revised the manuscript critically for important intellectual content. All the authors approved the final version to be published. HH, JK and EI are guarantors of this work.

Publisher's note Springer Nature remains neutral with regard to jurisdictional claims in published maps and institutional affiliations. 


\section{References}

1. Huxley RR, Filion KB, Konety S, Alonso A (2011) Meta-analysis of cohort and case-control studies of type 2 diabetes mellitus and risk of atrial fibrillation. Am J Cardiol 108(1):56-62. https://doi. org/10.1016/j.amjcard.2011.03.004

2. Fatemi O, Yuriditsky E, Tsioufis C et al (2014) Impact of intensive glycemic control on the incidence of atrial fibrillation and associated cardiovascular outcomes in patients with type 2 diabetes mellitus (from the Action to Control Cardiovascular Risk in Diabetes Study). Am J Cardiol 114(8):1217-1222. https://doi.org/10.1016/ j.amjcard.2014.07.045

3. Huxley RR, Alonso A, Lopez FL et al (2012) Type 2 diabetes, glucose homeostasis and incident atrial fibrillation: the Atherosclerosis Risk in Communities study. Heart 98(2):133-138. https://oi.org/10.1136/heartjnl-2011-300503

4. Chamberlain AM, Agarwal SK, Ambrose M, Folsom AR, Soliman EZ, Alonso A (2010) Metabolic syndrome and incidence of atrial fibrillation among blacks and whites in the Atherosclerosis Risk in Communities (ARIC) Study. Am Heart J 159(5):850-856. https:// doi.org/10.1016/j.ahj.2010.02.005

5. Scott RA, Scott LJ, Magi R et al (2017) An expanded genome-wide association study of type 2 diabetes in Europeans. Diabetes 66(11): 2888-2902. https://doi.org/10.2337/db16-1253

6. Scott RA, Lagou V, Welch RP et al (2012) Large-scale association analyses identify new loci influencing glycemic traits and provide insight into the underlying biological pathways. Nat Genet 44(9): 991-1005. https://doi.org/10.1038/ng.2385

7. Nielsen JB, Thorolfsdottir RB, Fritsche LG et al (2018) Biobankdriven genomic discovery yields new insight into atrial fibrillation biology. Nat Genet 50(9):1234-1239. https://doi.org/10.1038/ s41588-018-0171-3
8. Burgess S, Bowden J, Fall T, Ingelsson E, Thompson SG (2017) Sensitivity analyses for robust causal inference from Mendelian randomization analyses with multiple genetic variants. Epidemiology 28(1):30-42. https://doi.org/10.1097/EDE. 0000000000000559

9. Verbanck M, Chen CY, Neale B, Do R (2018) Detection of widespread horizontal pleiotropy in causal relationships inferred from Mendelian randomization between complex traits and diseases. Nat Genet 50(5):693-698. https://doi.org/10.1038/s41588-018-0099-7

10. Burgess S (2014) Sample size and power calculations in Mendelian randomization with a single instrumental variable and a binary outcome. Int J Epidemiol 43(3):922-929. https://doi.org/10.1093/ije/ dyu005

11. Chatterjee NA, Giulianini F, Geelhoed B et al (2017) Genetic obesity and the risk of atrial fibrillation: causal estimates from Mendelian randomization. Circulation 135(8):741-754. https:// doi.org/10.1161/CIRCULATIONAHA.116.024921

12. Jamaly S, Carlsson L, Peltonen M, Jacobson P, Sjostrom L, Karason K (2016) Bariatric surgery and the risk of new-onset atrial fibrillation in Swedish obese subjects. J Am Coll Cardiol 68(23): 2497-2504. https://doi.org/10.1016/j.jacc.2016.09.940

13. Larsson SC, Scott RA, Traylor M et al (2017) Type 2 diabetes, glucose, insulin, BMI, and ischemic stroke subtypes: Mendelian randomization study. Neurology 89(5):454-460. https://doi.org/ 10.1212/WNL.0000000000004173

14. Voight BF, Kang HM, Ding J et al (2012) The Metabochip, a custom genotyping array for genetic studies of metabolic, cardiovascular, and anthropometric traits. PLoS Genet 8(8):e1002793. https://doi.org/10.1371/journal.pgen.1002793 